\title{
Painfulness decreases the discriminability of electric shock
}

\author{
BILL JONES, MIGUEL PLANAS, and TERESA ANUZA \\ Carleton University, Ottawa, Ontario, Canada
}

\begin{abstract}
Two experiments were designed to investigate the relation between the reported painfulness and the discriminability of pairs of electric shocks for which the ratio between the higher and the lower intensity was held constant. In both experiments, subjects first gave category ratings of the painfulness of single shocks in the range .40 to $4.8 \mathrm{~mA}$. Then, in a discrimination experiment, they were required to identify the stronger of a pair of intensities presented in succession. The proportion of correct responses (Experiment 1) or the proportion of area under the receiver operating characteristic (Experiment 2) increased between a low-intensity pairing and a medium-intensity pairing and decreased between a medium-intensity pairing and a high-intensity pairing for subjects who rated the highest intensities as strongly painful. Subjects who gave moderate- or weak-pain ratings for the high-intensity shocks showed no significant increase or decrease in accuracy between the medium-and high-intensity pairings. Time-order errors were observed in Experiment 1. The proportion of trials on which the second stimulus of the pair was identified as the stronger increased monotonically as a function of stimulus magnitude. The results are discussed in the context of psychological assessment of analgesic procedures.
\end{abstract}

There are comparatively few psychophysical studies of painfulness (see, e.g., Rollman, 1977, or Sternbach, 1968, for reviews). Perhaps in consequence, psychophysical assessment of analgesic procedures is often dubious, at best (see, especially, Rollman, 1977). Several researchers (e.g., Chapman, Murphy, \& Butler, 1973) have argued that discriminability of pairs of painful stimuli is poorer if a potential analgesic (e.g., verbal suggestion, hypnosis, acupuncture, chemical agents, etc.) is effective. Conversely, a failure to find a change in an index of discriminability, such as the signal-detection index, $d^{\prime}$, has been taken to indicate the ineffectiveness of the analgesic. For example, Feather, Chapman, and Fisher (1972) found no change in $d^{\prime}$ for discrimination of levels of radiant heat after administration of an oral placebo and concluded that the placebo provided no relief from pain.

Rollman $(1977,1979)$ recently demonstrated the logical difficulties associated with this and similar arguments. He noted that an analgesic could diminish the painfulness of a pair of stimuli and yet have no effect on discriminability. Although Rollman specifically criticized some applications of signal-detection theory in the area of pain research, his point has a wider application (Jones, 1979). Discrimination of

Research made possible by a grant from the Medical Research Council of Canada to B. Jones and N. P. Spanos. A preliminary report of the research was given at the annual meeting of the Canadian Psychological Association, Toronto, 1981. The authors' complete mailing address is: Department of Psychology, Carleton University, Ottowa, Ontario K1S 5B6, Canada. painful stimuli, however indexed, can, in principle, increase, decrease, or remain constant whether or not an analgesic has an effect on painfulness.

Jones (1979) suggested that painfulness may in fact lower the discriminability of pairs of stimuli. It is generally agreed that the onset of pain gives rise to a complex set of physiological and psychological responses, including physical avoidance and implementation of strategies to cope with the pain (e.g., Spanos, Radtke-Bodorik, Ferguson, \& Jones, 1979). Appropriate response to the painfulness of a stimulus may therefore draw attention from the processing of information related to the task at hand. In a discrimination experiment, the result may be lower accuracy at painful intensities than at nonpainful ones.

Although Jones (1979) has claimed that some data of Rollman's (1979) possibly indicate higher intensity electric shocks are less well discriminated, there is little evidence for this proportion. Consequently, in the following experiments subjects were required to discriminate pairs of electric shocks that had been previously rated on a category scale of painfulness. If the ratio between the members of each stimulus pair is held constant, then the intensity difference must, of course, increase as intensity is increased. Under Weber's law, we expect, for equal ratios, discriminability to be constant across the stimulus range. However, it is known that subjects are also sensitive to stimulus differences. Petrusic and Jamieson (1978), for example, systematically varied both ratios and differences in a study of judgments of visual extent and showed that both variables were monotonic with 
subjects' judgment times. Therefore, as the difference in intensity between pairs of shocks is increased, we might expect a monotonic increase in discriminability. However, if painfulness affected discriminability, we would expect accuracy to decrease when shocks are perceived as painful.

\section{EXPERIMENT 1}

In this experiment, the subject was first required to rate the painfulness of unpaired electric shocks. A subset of the rated shocks was then used in a discrimination experiment. On any trial, the subject was presented with two shocks in succession and required to identify the more intense of the two. With the ratio of the higher to the lower intensity held constant at 1.075, the shock pairs were varied in intensity from typically nonpainful levels to typically moderately painful levels.

Two basic dependent measures were obtained in the discrimination experiment. The proportion of correct responses, $\mathrm{P}(\mathrm{C})$, provided an index of accuracy for the forced-choice experiment, and the proportion of trials on which the second stimulus was identified as the more intense, $\mathrm{P}(2)$, provided an index of the so-called time-order error (TOE). TOEs, first noted by Fechner (1966) in an experiment on the discrimination of lifted weights, seem to be a highly consistent feature of perceptual judgments of pairs of stimuli (e.g., Jamieson \& Petrusic, 1975a, 1975b). In Fechner's terminology, a positive TOE refers to a tendency to judge the first presented stimulus as the more intense, that is, $P(2)<.5$, and a negative TOE refers to a tendency to judge the second stimulus as the more intense, that is, $P(2)>.5$. In a previous study of the painfulness of pairs of shocks, Geertsma (1958) found that the direction of the TOE was a function of the duration of the interstimulus interval (ISI).

\section{Method}

Subjects. Forty-eight subjects took part in the experiment for payment of $\$ 10 / \mathrm{h}$. All were aware in advance that the experiment would involve painful electric shocks, and all were free to withdraw at any point.

Apparatus. A Lafayette device delivered ac pulses through concentric electrodes (Tursky, Watson, \& O'Connell, 1965) into an electric skin circuit that maintained impedance at 5,000 $500 \Omega$. The equipment met CSA standards for safety. The duration of shocks and the interval between shocks were controlled by locally constructed timing equipment.

Procedure. The subject was seated on a stool and two electrodes were attached to the volar surface of the left forearm. In the first phase of the experiment, the experimenter acquainted the subject with the maximum stimulus range by presenting, in an ascending order, single shocks, from $.40 \mathrm{~mA}$ to $4.80 \mathrm{~mA}$ in intensity, through a single electrode. At this point, the subjects were asked if they wished to continue. All 48 subjects did so. The subject was then required to rate the painfulness of each shock using the following numerical scale: $1=$ no sensation, $2=$ tactile sensation, $3=$ very faint pain, $4=$ faint pain, $5=$ mild pain, $6=$ moderate pain, $7=$ strong pain, $8=$ very strong pain. Fifteen shocks in the range were presented twice in a random order, and the subject rated each shock according to the scale.

In the final phase of the experiment, three pairs of shocks from the range were used in the discrimination experiment. On each trial, two shocks, each $500 \mathrm{msec}$ in duration, were presented in sequence, one over each electrode and with an interstimulus interval of $250 \mathrm{msec}$. The subject was required to report whether the more intense of the two was "first" or "second" in the sequence. The stimulus pairs were .80 and $.86 \mathrm{~mA}, 2.4$ and $2.58 \mathrm{~mA}$, and 4.25 and $4.57 \mathrm{~mA}$. For a block of 40 trials, one pair of shocks was used and the order in which the shocks were presented was randomized over trials. Whether the first shock was presented over one electrode or the other was also randomized. The order in which blocks of trials were presented was randomized across subjects.

\section{Results}

The means and standard deviations for $P(C)$ and $P(2)$ and the painfulness ratings are shown in Table 1. The ratings are averaged across four presentations, two presentations of the lower intensity and two of the higher. The low-intensity pair $(.80 / .86 \mathrm{~mA})$ was typically rated as nonpainful, the medium-intensity pair $(2.4 / 2.58 \mathrm{~mA})$ as faintly painful, and the highintensity pair $(4.25 / 4.57 \mathrm{~mA})$ as moderately painful. For all but one of the 48 subjects, the ratings were monotonic with stimulus intensity.

It is apparent that the TOE was a function of stimulus intensity. TOE was positive for the low intensities, zero for the medium intensities, and negative for the high intensities. A one-way analysis of variance showed that the effect of intensities was significant $[F(2,94)=40.19, p<.0001]$. The NewmanKeuls procedure showed that all three means differed significantly from each other at the .01 level.

In line with our prediction that painfulness should affect discriminability, $P(C)$ values increased for the moderate-intensity shocks but decreased for the higher intensities. Again, a one-way analysis of variance showed a significant effect of stimulus intensity $[F(2,94)=13.14, p<.001]$, and Newman-Keuls showed that the three means differed significantly from each other.

This result corresponds to Holway and Pratt's (1938) classic demonstration that the Weber fraction may be higher for high-stimulus intensities, which in the present experiment were typically associated with reports of moderate painfulness. To make the case that the decrease in discriminability is related to painfulness, and not simply to higher stimulus intensities, we need to show that discriminability

Table 1

$\mathbf{P}(C), P(2)$, and Mean Ratings for the Three Intensity Levels

\begin{tabular}{|c|c|c|c|c|c|c|}
\hline & \multicolumn{6}{|c|}{ Intensity Level } \\
\hline & \multicolumn{2}{|c|}{ Low } & \multicolumn{2}{|c|}{ Medium } & \multicolumn{2}{|c|}{ High } \\
\hline & Mean & SD & Mean & $\mathrm{SD}$ & Mean & SD \\
\hline $\begin{array}{l}\mathrm{P}(\mathrm{C}) \\
\mathrm{P}(2) \\
\text { Ratings }\end{array}$ & $\begin{array}{r}.68 \\
.44 \\
1.75 \\
\end{array}$ & $\begin{array}{l}.15 \\
.12 \\
.81 \\
\end{array}$ & $\begin{array}{r}.79 \\
.50 \\
4.27 \\
\end{array}$ & $\begin{array}{r}.11 \\
.11 \\
1.52 \\
\end{array}$ & $\begin{array}{r}.74 \\
.63 \\
6.09 \\
\end{array}$ & $\begin{array}{r}.10 \\
.12 \\
1.45 \\
\end{array}$ \\
\hline
\end{tabular}


of the high-intensity pair is a function of pain ratings. The correlation between $P(C)$ for the highintensity pair and pain ratings was -.24 , which is significant at the .05 level [ $t(46)=1.69$ ]. In other words, $P(C)$ tended to decrease as reported painfulness increased.

To illustrate this point, subjects were classified as strong-, moderate-, and weak-pain raters on the following basis. For strong-pain raters, the mean ratings of shocks in the high-intensity pair was at least 7 (strong pain). For moderate raters, the mean rating for the same pair was greater than 5 and less than 7 (mild to moderate pain), and for weak raters, the mean rating was less than or equal to 5 (faint to mild pain). Some statistical characteristics of the three subject groups are shown in Table 2.

Mean $P(C)$ values for the three subject groups are shown in Figure 1 as a function of stimulus difference. The figure provides a clear illustration of our hypothesis. Weak-pain ratings were associated with a monotonic increase in $P(C)$ as the stimulus difference increased. There was a moderate decrease in accuracy between the high- and medium-intensity pairs for moderate-pain ratings and a steep decline between the same points for strong-pain ratings. A two-way analysis of variance using a least squares solution for the effect of unequal group size showed a significant effect of stimulus intensity $[F(2,90)=15.47, p<.0001]$ and a significant subject group $\times$ stimulus intensity interaction $[F(4,90)=5.20, p<.01] . P(C)$ decreased significantly $(p<.01)$ between medium and high intensities in the strong-pain group and did not increase or decrease significantly between the same intensities in the moderate- and weak-pain groups (NewmanKeuls).

Interestingly, the decrease at the high intensity for the subjects who gave strong-pain ratings was not relative to increased accuracy for this group at the medium intensity. The Newman-Keuls procedure showed that the three subject groups were statistically equivalent in accuracy of discrimination of the medium-intensity shocks.

For all three groups, the TOE increased monotonically as a function of stimulus magnitude (Figure 2), and a two-way analysis of variance showed no effect of subject group and no interaction between subject groups and stimulus intensities.

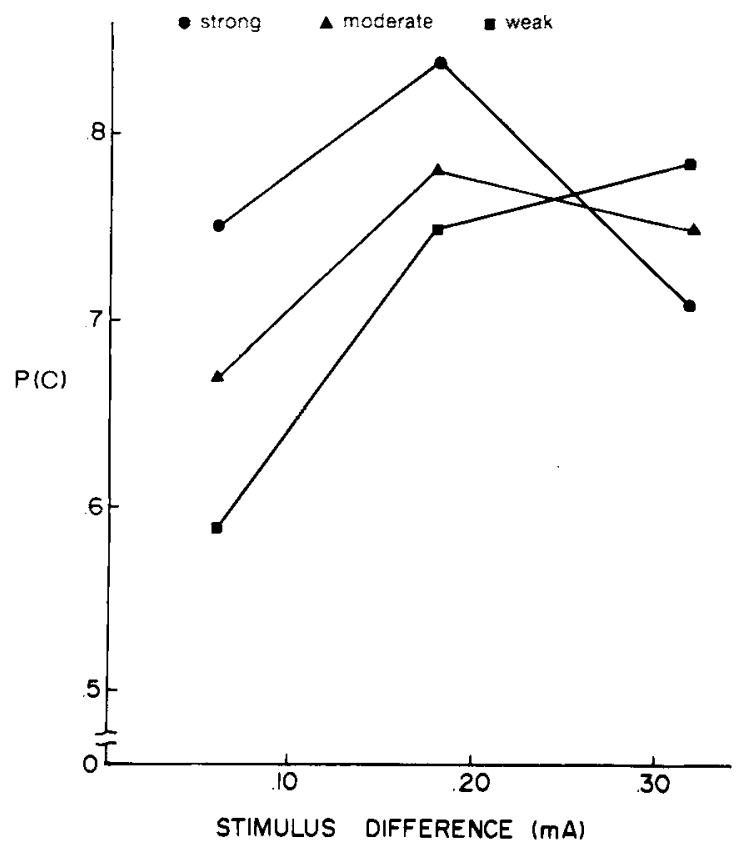

Figure 1. Mean $P(C)$ values as a function of the difference between shock pairs for strong-, moderate-, and weak-pain raters (Experiment 1).

\section{EXPERIMENT 2}

Although the TOE is not well understood, there is some reason to believe that the effect is not due to a simple response bias in the signal-detection sense (see Jamieson \& Petrusic, 1975a). However, the index of TOE, $P(2)$, can in principle be regarded as an index of bias, and it is well known that, as bias increases, $P(C)$ tends to decrease (Green \& Swets, 1966). One might argue, therefore, that the decrease in $P(C)$ shown by strong-pain raters merely reflects an increase in response bias. This account would be difficult to sustain, because $P(2)$ increased between the medium- and high-intensity shocks for all subject groups, whereas a decrease in $P(C)$ was associated only with strong-pain ratings. Nevertheless, in the following experiment we obtained, by means of receiver-operating-characteristic ( $R O C)$ analysis, indices of accuracy of discrimination independent of response bias.

Table 2

Rating Data for the Three Intensity Levels by Each Subject Group (Experiment 1)

\begin{tabular}{|c|c|c|c|c|c|c|c|c|c|c|}
\hline \multirow[b]{3}{*}{ Pain Ratings } & \multirow[b]{3}{*}{$\mathrm{n}$} & \multicolumn{9}{|c|}{ Intensity Level } \\
\hline & & \multicolumn{3}{|c|}{ Low } & \multicolumn{3}{|c|}{ Medium } & \multicolumn{3}{|c|}{ High } \\
\hline & & Mcan & SD & Range & Mean & SD & Range & Mean & SD & Range \\
\hline Weak & 10 & 1.30 & .37 & $1.00-1.25$ & 2.73 & .58 & $2.00-3.75$ & 3.85 & .87 & $2.00-4.75$ \\
\hline Moderate & 22 & 1.57 & .44 & $1.00-2.00$ & 3.81 & .71 & $2.75-4.75$ & 6.02 & .49 & $5.25-6.75$ \\
\hline Strong & 16 & 2.28 & 1.07 & $1.00-4.00$ & 5.88 & 1.26 & $2.00-6.75$ & 7.59 & .36 & $7.00-8.00$ \\
\hline
\end{tabular}




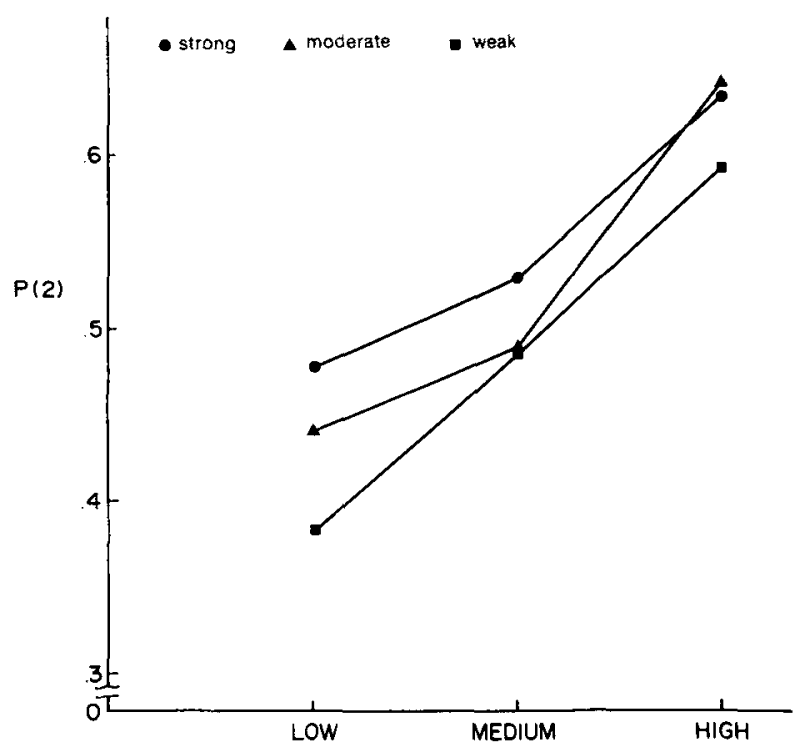

STIMULUS MAGNITUDE

Figure 2. Mean P(2) values, the TOE, as a function of the magnitude of the shock pairs for strong-, moderate-, and weak-pain raters (Experiment 1).

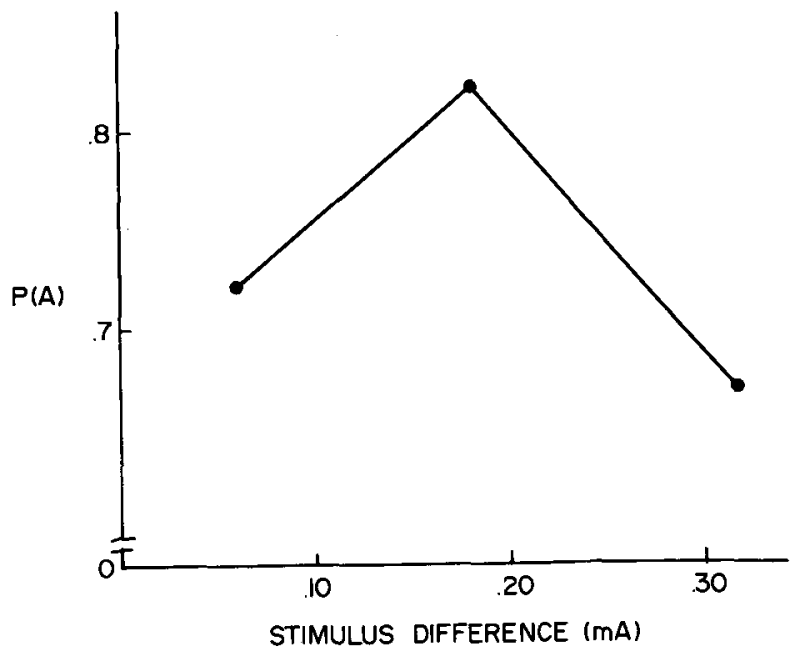

Figure 3. Mean $P(A)$ values as a function of the difference between shock pairs (Experiment 2).

\section{Method}

Subjects. The subjects were seven volunteers who were paid at a rate of $\$ 10 / \mathrm{h}$. All seven subjects had rated the high-intensity stimulus pair at 7 or above.

Apparatus and Procedure. The apparatus was the same as in Experiment 1. The procedure was also the same, except that in the discrimination phase the number of trials was increased to 60 per block and the subject was required to respond using a numerical rating scale with categories 1 = certain "first," 2 =probably "first," 3 = possibly "first," 4 = possibly "second," $5=$ probably "second," $6=$ certain "second," which indicated the degree of confidence that the more intense shock was first or second in the sequence.

\section{Results}

ROC curves were constructed from the rating-scale data for each subject at each of three intensity levels. The index of discriminability was the proportion of area under the ROC curve, $\mathrm{P}(\mathrm{A})$, a distribution-free index of accuracy independent of response bias (Green \& Swets, 1966). Mean values of $P(A)$ as a function of stimulus differences are shown in Figure 3. It is apparent that these data conform closely to the data in Figure 1. A one-way analysis of variance showed a significant effect of stimulus intensity $[F(2,12)=$ $=4.05, \mathrm{p}<.05]$, and a Newman-Keuls test showed a significant $(p<.05)$ decrease in $P(A)$ between the medium- and high-intensity pairs. Given this result and the data from Experiment 1, it is difficult to argue that the decrease in $P(C)$ in the two experiments resulted from a simple response bias. Rather, painfulness appears to have a direct effect on discriminability.

\section{CONCLUSIONS}

Both experiments demonstrated a clear relationship between reported painfulness and the accuracy with which pairs of shocks were discriminated. Although the high-intensity stimuli differed by the greatest amount, discriminability tended to decrease significantly between this pair and the medium-intensity pair, and this effect was related to individual differences in pain ratings. A significant decrement was shown only by those subjects who gave strong-pain ratings.

To some it may be intuitively obvious that experience of pain should detrimentally affect discriminability, although, as we have noted, many researchers have apparently believed that relief from pain should be associated with poorer discrimination. In the light of that position, the interest of the present data is largely normative. In ordinary circumstances, we may expect subjects to have difficulty discriminating stimuli that they have described as markedly painful. Any assessment of an analgesic procedure must take this effect into account. However, there is almost certainly no single experiment or no single statistic that will unequivocally demonstrate that subjects have obtained genuine relief from pain.

\section{REFERENCES}

Chapman, C. R., Murphy, J. M., \& Butlen, S. H. Analgesic strength of $33 \%$ nitrous oxide: A signal detection theory evaluation. Science, 1973, 179, 1246-1248.

Feather, B. W., Chapman, C. R., \& Fisher, S. B. The effect of a placebo on the perception of painful radiant heat stimuli. Psychosomatic Medicine, 1972, 34, 290-294.

Fechner, G. [Elements of psychophysics] (H. E. Adler, trans.) New York: Holt, 1966.

Gentsma, R. H. Time-order errors in comparative judgements of hurtfulness. Journal of Experimental Psychology, 1958, 55, 284-288. 
Green, D. M., \& Swets, J. A. Signal detection theory and psychophysics. New York: Wiley \& Sons, 1966.

Holway, A. H., \& Pratt, C. C. The Weber-ratio for intensive discrimination. Psychological Review, 1938, 45, 430-434.

Jamieson, D. G., \& Petrusic, W. M. The dependence of timeorder error direction on stimulus range. Canadian Journal of Psychology, 1975, 29, 175-182. (a)

Jamieson, D. G., \& Petrusic, W. M. Pairing effects and timeorder errors in duration discrimination. Perception \& Psychophysics, 1975, 18, 107-113. (b)

Jones, B. Signal detection theory and pain research. Pain, 1979, 7, 305-312.

Petrusic, W. M., \& Jamieson, D. G. Resolution time and the coding of arithmetic relations on supraliminally different visual extents. Journal of Mathematical Psychology, 1978, 10, 89-107.

Rollman, G. B. Signal detection theory measurement of pain: A review and critique. Pain, 1977, 3, 187-211.
Rollman, G. B. Signal detection theory pain measures: Empirical validation studies and adaptation-level effects. Pain, 1979, 6, 9-21.

Spanos, N. P., Radtke-Bodorik, H. L., Ferguson, J. D., \& JoNES, B. The effects of hypnotic susceptibility, suggestions for analgesia and the utilization of cognitive strategies on the reduction of pain. Journal of Abnormal Psychology, 1979; 88, 282-292.

Sternbach, R. A. Pain: A psychophysical analysis. New York: Academic Press, 1968.

Tursky, B., Watson, P. D., \& O'Connell, D. N. A concentric shock electrode for pain stimulation. Psychophysiology, 1965, 1, 269-298.

(Manuscript received June 6, 1981;

revision accepted for publication March 23, 1982.) 